\title{
The clinical significance of collagen family gene expression for esophageal squamous cell carcinoma
}

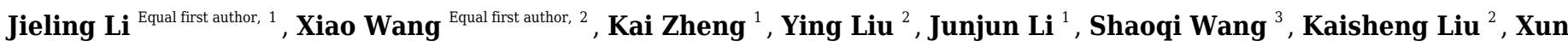 \\ Song ${ }^{1}$, Nan $\mathrm{Li}^{2}$, Shouxia Xie ${ }^{\text {Corresp., }}{ }^{2}$, Shaoxiang Wang ${ }^{\text {Corresp. } 1}$ \\ ${ }^{1}$ School of Pharmaceutical Sciences, Shenzhen University Health Science Center, Shenzhen, China \\ Department of Pharmacy, The Second Clinical Medical College (Shenzhen People's Hospital), Jinan University, Shenzhen, China \\ 3 Department of Oncology, Hubei Provincial Corps Hospital, Chinese People Armed Police Forces, Wuhan, China \\ Corresponding Authors: Shouxia Xie, Shaoxiang Wang \\ Email address: szshouxia@163.com,wsx@szu.edu.cn
}

Background: Esophageal squamous cell carcinoma (ESCC) is a subtype of esophageal cancer with high incidence and mortality. Due to the poor five-year survival rates of patients with ESCC, exploring novel diagnostic markers for early ESCC is emergent. Collagen, the abundant constituent of extracellular matrix, plays a critical role in tumor growth and epithelial-mesenchymal transition. However, the clinical significance of collagen genes in ESCC has been rarely studied. In this work, we systematically analyzed the gene expression of whole collagen family in ESCC, aiming to search for ideal biomarkers.

Methods: Clinical data and gene expression profiles of ESCC patients were collected from The Cancer Genome Atlas (TCGA) and the Gene Expression Omnibus (GEO) databases. Bioinformatics methods, including differential expression analysis, survival analysis, gene sets enrichment analysis (GSEA) and coexpression network analysis, were performed to investigate the correlation between the expression patterns of 44 collagen family genes and the development of ESCC.

Results: 22 genes of collagen family were identified as differentially expressed genes (DEGs) in both the two datasets. Among them, COL1A1, COL10A1 and COL11A1 were particularly up-regulated in ESCC tissues compared to normal controls, while COL4A4, COL6A5 and COL14A1 were notably down-regulated. Besides, patients with low COL6A5 expression or high COL18A1 expression showed poor survival. In addition, a 7-gene prediction model was established based on collagen gene expression to predict patient survival, which had better predictive accuracy than the tumor-node-metastasis (TNM) staging based model. Finally, GSEA results suggested that collagen genes might be tightly associated with $\mathrm{PI3K/Akt/mTOR} \mathrm{pathway,} \mathrm{p53} \mathrm{pathway,} \mathrm{apoptosis,} \mathrm{cell} \mathrm{cycle,} \mathrm{etc.}$

Conclusion: Several collagen genes could be potential diagnostic and prognostic biomarkers for ESCC. Moreover, a novel 7-gene prediction model is probably useful for predicting survival outcomes of ESCC patients. These findings may facilitate early detection of ESCC and help improves prognosis of the patients. 


\section{The clinical significance of collagen family gene expression for esophageal}

\section{2 squamous cell carcinoma}

3

4 Jieling $\mathrm{Li}^{1 *}$, Xiao Wang ${ }^{2 *}$, Kai Zheng ${ }^{1}$, Ying $\operatorname{Liu}^{2}$, Junjun $\mathrm{Li}^{1}$, Shaoqi Wang ${ }^{3}$, Kaisheng Liu ${ }^{2}$, Xun

5 Song ${ }^{1},{\mathrm{Nan} \mathrm{Li}^{2} \text {, Shouxia Xie}}^{2}$, Shaoxiang Wang ${ }^{1}$

6

$7{ }^{1}$ School of Pharmaceutical Sciences, Shenzhen University Health Science Center, Shenzhen, 8 China

9 2Department of Pharmacy, The Second Clinical Medical College (Shenzhen People's Hospital), 10 Jinan University, Shenzhen, China

$11{ }^{3}$ Department of Oncology, Hubei Provincial Corps Hospital, Chinese People Armed Police Forces, 12 Wuhan, China

$13{ }^{*}$ These authors contributed equally to this work.

15 Corresponding authors:

16 Shaoxiang Wang ${ }^{1}$

17 Email address: wsx@szu.edu.cn

18 Shouxia Xie ${ }^{2}$

19 Email address: szshouxia@163.com 


\section{Abstract}

Background: Esophageal squamous cell carcinoma (ESCC) is a subtype of esophageal cancer with high incidence and mortality. Due to the poor five-year survival rates of patients with ESCC, exploring novel diagnostic markers for early ESCC is emergent. Collagen, the abundant constituent of extracellular matrix, plays a critical role in tumor growth and epithelialmesenchymal transition. However, the clinical significance of collagen genes in ESCC has been rarely studied. In this work, we systematically analyzed the gene expression of whole collagen family in ESCC, aiming to search for ideal biomarkers.

Methods: Clinical data and gene expression profiles of ESCC patients were collected from The Cancer Genome Atlas (TCGA) and the Gene Expression Omnibus (GEO) databases. Bioinformatics methods, including differential expression analysis, survival analysis, gene sets enrichment analysis (GSEA) and co-expression network analysis, were performed to investigate the correlation between the expression patterns of 44 collagen family genes and the development of ESCC.

Results: 22 genes of collagen family were identified as differentially expressed genes (DEGs) in both the two datasets. Among them, COL1A1, COL10A1 and COL11A1 were particularly upregulated in ESCC tissues compared to normal controls, while COL4A4, COL6A5 and COL14A1 were notably down-regulated. Besides, patients with low COL6A5 expression or high COL18A1 expression showed poor survival. In addition, a 7-gene prediction model was established based on collagen gene expression to predict patient survival, which had better predictive accuracy than the tumor-node-metastasis (TNM) staging based model. Finally, GSEA results suggested that collagen genes might be tightly associated with PI3K/Akt/mTOR pathway, p53 pathway, apoptosis, cell cycle, etc.

Conclusion: Several collagen genes could be potential diagnostic and prognostic biomarkers for ESCC. Moreover, a novel 7-gene prediction model is probably useful for predicting survival outcomes of ESCC patients. These findings may facilitate early detection of ESCC and help improves prognosis of the patients.

\section{Introduction}

Esophageal cancer is the seventh most commonly diagnosed cancer and the sixth leading cause of cancer death (Bray et al. 2018). It is classified into two histological subtypes, esophageal adenocarcinoma (EAC) and esophageal squamous cell carcinoma (ESCC), the latter of which is the predominant type worldwide (Pennathur et al. 2013). Despite the effective treatments (e.g. surgery, chemotherapy and radiotherapy) for ESCC, the 5-year survival rates of patients with advanced ESCC are still less than 20\% (Codipilly et al. 2018). However, the survival rates could be improved to over $80 \%$ if patients were diagnosed with an early stage (Lao-Sirieix \& Fitzgerald 2012; Wang et al. 2004). Although a few tumor markers, carcinoembryonic antigen (CEA), carbohydrate antigen (CA) 19-9, and squamous cell carcinoma (SCC) antigen, have been used in the diagnosis of ESCC, they are not suitable for early detection due to the lack of sensitivity 
59 (Kosugi et al. 2004). Thus, it is urgent to search for novel biomarkers to help early detection of 60 ESCC and improve survival rates of the patients.

61 Collagen is the most abundant extracellular matrix protein that promotes cell growth and family comprises 28 types with different $\alpha$ Chains encoded by more than 40 genes (Ricard-Blum 2011). It has been reported that the expression of collagen-encoding genes was significantly related to the prognosis of certain types of cancers (Giussani et al. 2018; Liu et al. 2018; Rong et al. 2018; Shen et al. 2016; Zhang et al. 2018c). In addition, a couple of collagen genes, such as COL11A1 and COL6A1, were expressed aberrantly in ESCC tissues and possibly affected the progression of ESCC (Fan et al. 2012; He et al. 2017; Zhang et al. 2018a). However, most of these works focused on specific collagen genes, and the potential roles of other members remain to be clarified.

Here we provided a systematic analysis of gene expression of the whole collagen family and its corresponding clinical significance in ESCC. Clinical data and gene expression profiles of ESCC patients were extracted from The Cancer Genome Atlas (TCGA) and the Gene Expression Omnibus (GEO), two public databases with substantial information about cancers. Different bioinformatics methods, including differential expression analysis, survival analysis, pathway analysis and co-expression network analysis were used to analyze the data to sift important hits possibly involved in the initiation and development of ESCC. According to collagen family genes, we also established a prediction model with high performance to predict the prognosis of ESCC patients. Collectively, our works mainly explored the relation of collagen gene expression to ESCC and illuminated the potential mechanism.

\section{Materials \& Methods}

\section{Patient data}

Basic data of ESCC patients were downloaded from the TCGA database (https://portal.gdc.cancer.gov/) and the GSE53625 dataset of the GEO database (https://www.ncbi.nlm.nih.gov/geo/query/acc.cgi?acc=GSE53625), 95 cases from TCGA and 179 cases from GSE53625. Univariate and multivariate Cox regression analyses were carried out to investigate the correlation between overall survival and clinicopathological characteristics of the patients by SPSS (v23.0). The relations between collagen family gene expression and clinicopathological characteristics of the patients were examined using Pearson correlation analysis via SPSS.

\section{Differential expression analysis}

Gene expression profiles of tumor and adjacent normal tissues in ESCC patients were also obtained from the two datasets. 81 of 95 patient cases in TCGA and all patient cases in GEO had RNAsequence data. In total, 81 tumor samples with 11 normal controls from TCGA and 179 tumor samples with 179 normal controls from GEO (Li et al. 2014) were included in analysis (each sample was taken from a different patient). Differential expression analysis was conducted using the edgeR (Robinson et al. 2010) and the limma (Ritchie et al. 2015) packages respectively for 
TCGA and GEO data by R software (https://www.r-project.org/, v3.5.3). Gene expression levels were normalized by the calcNormFactors function in edgeR (Law et al. 2016) and by the normalizeBetweenArrays function in limma (Smyth \& Speed 2003), to make the expression distributions of each sample are similar across the entire matrix. Then based on the exact test in edgeR which is analogous to Fisher's exact test (Robinson et al. 2010) and the Empirical Bayes statistical test in limma (Phipson et al. 2016), fold change (FC), $P$ value and false discovery rate (FDR) (or adjusted $P$ value) were figured out to show the expression difference between tumor and normal samples. Genes with $P<0.05$ and FDR $<0.05$ were considered as differentially expressed genes (DEGs). Accordingly, DEGs of collagen family were identified. Then heatmaps, boxplots and Venn diagram were drawn by R software.

\section{Survival analysis}

First, hazard ratio (HR) and $P$ value of each DEG of collagen family were figured out based on gene expression and overall survival of patients by the univariate Cox regression model with the survival package through R software. The HR is an estimate of the ratio of the hazard rate in the treated versus the control group (Spruance et al. 2004), while in this study it is defined as the hazard in the high expression group divided by the hazard in the low expression group. HR $>1$ and $\mathrm{HR}<1$ mean higher expression of the gene is associated with worse and better overall survival respectively. Survival curves were plotted according to the Kaplan-Meier method and compared by the log-rank test using the survival and the qvalue packages in R. $P<0.05$ was considered statistically significant.

\section{Prediction models}

Prediction models were established to predict patient survival based on gene expression of 22 DEGs of collagen family and overall survival of patients by the multivariate Cox regress analysis with the survival package via R software. Several candidate genes were eventually selected out by the analysis to form the model, with a formula calculating the risk score of each patient. The general formula is given below:

$$
\text { Risk score }=\sum_{i=1}^{n} \operatorname{Coef}_{i} \times \operatorname{Exp}_{i}
$$

where n, Coef, and Exp indicate the number of included genes, the coefficient of each gene, and gene expression level, respectively. The coefficients were estimated based on the relative contributions of each collagen gene. A patient's risk score was calculated as the sum of the expression levels of each gene multiplied by its corresponding coefficient. Similar methods have been adopted by earlier studies (Beer et al. 2002; Lossos et al. 2004; Wang et al. 2018). Then receiver operating characteristic (ROC) curves were plotted based on the risk scores and overall survival of patients by the survivalROC package in R, with area under curve (AUC) values which represented the accuracy of predicting 3-year survival. Also, survival curves were obtained by dividing the patients into high- and low-risk groups according to the median risk score using the survival package. 


\section{Pathway analysis}

Potential mechanism of collagen family genes was explored by the gene sets enrichment analysis (GSEA), a method to determine whether members of a previously defined gene set are correlated with the phenotypic class distinction (Subramanian et al. 2005). GSEA was conducted using the gene expression profiles of patients' tumor samples via javaGSEA software (http://software.broadinstitute.org/gsea/downloads.jsp), and the patient samples were divided into high- and low-risk groups in half according to the risk scores obtained by the collagen-DEGsbased prediction models (Chai et al. 2018; Zhang et al. 2017; Zhao et al. 2017). Oncogenic Signatures Gene Sets (v6.2), Hallmark Gene Sets (v6.2) and KEGG Gene Sets (v6.2) (http://software.broadinstitute.org/gsea/msigdb/collections.jsp) were respectively used as references. Based on these gene sets databases, the expression profiles were analyzed to find out if a set of genes were mostly up-regulated (or down-regulated) in the high-risk group (or low-risk group). Normalized enrichment score (NES) reflected the degree to which a gene set was overrepresented in the groups, and gene sets in the results with $\mathrm{P}<0.05$ and FDR $<0.25$ were considered as significant ones (Subramanian et al. 2005).

\section{Co-expression network analysis}

Patients' tumor samples from TCGA were separated into high- and low-risk groups by the risk scores calculated by the 7-gene prediction model. Risk-score-based DEGs that were differentially expressed between the two groups were determined using the gene expression profiles of tumor samples by the same method as differential expression analysis. Then the relationships between collagen family genes and the risk-score-based DEGs as well as the representative enriched gene sets from GSEA were assessed by the Weighted Gene Co-Expression Network Analysis (WCGNA) with the WGCNA package through R software, which is a method to describe the correlation patterns among genes across different samples (Langfelder \& Horvath 2008). Genes of each gene set were extracted from http://software.broadinstitute.org/gsea/msigdb/genesets.jsp. Finally, the genes co-expressed with collagen family genes were obtained, and the networks of them were drawn via Cytoscape (http://www.cytoscape.org/, v3.7.1).

\section{Results}

\section{Clinicopathological information of the ESCC patients}

A total of 95 patient cases in TCGA and 179 cases in GEO were collected and analyzed by univariate and multivariate Cox regression analyses. As a result, poor overall survival was significantly correlated with sex, TNM stage and N stage in TCGA $(P=0.020, P=0.015$, and $P$ $=0.012$, respectively) (Table 1 ), and was notably associated with age, TNM stage and N stage in GEO ( $P=0.021, P<0.001$, and $P=0.030$, respectively) (Table 2$)$. Besides, investigation into the correlation between collagen family gene expression and the clinicopathological characteristics revealed that the expression of several collagen genes was significantly related to advanced TNM stages or tumor grades. (Table 3 and Table 4). 
179

180

181

182

183

184

185

186

187

188

189

190

191

192

193

194

195

196

197

198

199

200

201

202

203

204

205

206

207

208

209

210

211

212

213

214

215

216

217

218

219

\section{Identification of DEGs of collagen family in ESCC tissues}

Differential expression analysis showed that more than $2 / 3$ of the 44 collagen family genes were up-regulated in tumor tissues in both TCGA and GEO (Tables S1 and S2). 22 members in TCGA and 35 members in GEO were identified as DEGs, and their expression patterns were shown by heatmaps (Figs. 1A and 1B). Then the Venn diagram demonstrated that there were 22 mutual DEGs between the two datasets (Fig. 1C), which meant the DEGs observed in TCGA were also DEGs in GEO. Obviously from the heatmaps, COL1A1, COL10A1 and COL11A1 ranked in the top five among the up-regulated DEGs in both datasets (Figs. 1D-1I), further presented by boxplots. Likewise, COL4A4, COL6A5 and COL14A1 were the most down-regulated candidates (Figs. 1J-1O).

\section{Survival analysis of collagen family genes in ESCC patients}

HRs and $P$ values of the 22 DEGs were calculated and shown by heatmaps (Figs. 2A and 2B). Among them, HRs of COL6A5 and COL18A1 were the lowest and highest respectively. Survival curves of the DEGs were plotted according to the Kaplan-Meier method. Consistently, COL6A5 and COL18A1 were the two genes most relevant to the overall survival of ESCC patients. Patients with lower COL6A5 expression exhibited poorer overall survival $(P=0.008$ in TCGA, Fig. 2C; $P$ $=0.060$ in GEO, Fig. 2D). By contrast, patients with higher COL18A1 expression had worse overall survival ( $P=0.393$ in TCGA, Fig. $2 \mathrm{E} ; P=0.009$ in GEO, Fig. 2F). These results suggested that COL6A5 and COL18A1 are tightly associated with the prognosis of ESCC.

\section{DEGs-based prediction models to predict the prognosis of ESCC patients}

ROC curves have been extensively used to evaluate the predictive effect of one or more genes. The AUC value represents predictive accuracy and usually makes sense when it exceeds 0.60 (Ludemann et al. 2006; Metz 1978; Obuchowski 2003). ROC curves of COL6A5 and COL18A1 indicated that good predictive performance could only be attained by COL6A5 in TCGA (AUC=0.679, Fig. S1A), while COL18A1 had no predictive ability (Figs. S1C and S1D), suggesting that a single gene is not suitable for survival prediction of ESCC patients. Therefore, we established multi-gene prediction models based on expression levels of the DEGs to assess the joint effect of selected collagen genes on patient survival. There were 7 genes in TCGA and 9 genes in GEO finally included to form the models respectively, and risk scores of the patients were calculated according to the below formulas:

Risk score $($ TCGA $)=\left(1.528 *\right.$ COL1A $\left.1_{\text {Exp }}\right)+\left(0.265 *\right.$ COL4A4 $\left.4_{\text {Exp }}\right)+\left(-0.539 * \operatorname{COL} 6 A 5_{\text {Exp }}\right)+(-$ $0.638 *$ COL11A $\left.1_{\text {Exp }}\right)+\left(-1.193 * \operatorname{COL} 12 A 1_{\text {Exp }}\right)+\left(-0.244 * \operatorname{COL} 19 A 1_{\text {Exp }}\right)+\left(0.417 * \operatorname{COL} 24 \mathrm{~A} 1_{\text {Exp }}\right)$.

Risk score $(\mathrm{GEO})=\left(7.700 * \mathrm{COL} 1 \mathrm{~A} 1_{\text {Exp }}\right)+\left(-8.800 * \operatorname{COL} 1 \mathrm{~A} 2_{\text {Exp }}\right)+\left(-5.800 * \mathrm{COL} 3 \mathrm{~A} 1_{\text {Exp }}\right)+$ $\left(6.320 * \operatorname{COL} 5 \mathrm{~A} 1_{\operatorname{Exp}}\right)+\left(-0.708 * \operatorname{COL} 6 \mathrm{~A} 5_{\operatorname{Exp}}\right)+\left(-0.790 * \mathrm{COL} 11 \mathrm{~A} 1_{\operatorname{Exp}}\right)+\left(1.990 * \mathrm{COL} 14 \mathrm{~A} 1_{\text {Exp }}\right)+$ $\left(1.300 *\right.$ COL22A $\left.1_{\text {Exp }}\right)+\left(2.400 *\right.$ COL24A $\left.1_{\text {Exp }}\right)$.

For instance, the positive coefficient for COL1A1 suggests that higher expression of COL1A1 was associated with worse survival. The negative value allocated to COL6A5 means that higher expression of COL6A5 was related to prolonged survival, in agreement with the survival analysis 
220

221

222

223

224

225

226

227

228

229

230

231

232

233

234

235

236

237

238

239

240

241

242

243

244

245

246

247

248

249

250

251

252

253

254

255

256

257

258

259

260

(Fig. 2). Notably, AUCs on the ROC curves of the DEGs-based models in TCGA and GEO reached 0.86 and 0.68 respectively (Figs. $3 \mathrm{~A}$ and $3 \mathrm{C}$ ), which were higher than those of the prediction models based on TNM staging in the two datasets with AUCs of 0.625 and 0.646 respectively (Figs. 3E and 3G). The TNM staging system is a generally recognized standard for classifying the spreading extent of cancer (D'Journo 2018) and is commonly used to predict prognosis of cancer in clinical application. The prediction models respectively based on T-stage and N-stage were also examined but the AUCs were all less than 0.6 (Fig. S2). Furthermore, survival curves showed that patients with high risk were significantly correlated with poor survival (Figs. 3B, 3D, 3F and 3H). The 7-gene model in TCGA with true positive rate of $86 \%$ was more accurate than that of the TNM staging-based model, whereas predictive accuracy of the 9-gene model in GEO exhibited no difference. Therefore, the model in TCGA was used for our further studies. Finally, a heatmap was plotted to show the expression patterns of the 7 genes in TCGA between high-risk and low-risk groups (Fig. 3I). The risk score distribution was exhibited in ascending order, and patients were divided into high- and low-risk groups by the median point (Fig. 3J). Overall, it can be seen that patients with high risk score had higher mortality rates and shorter survival time than those with low risk score (Fig. 3K). Taken together, above results indicated that the 7-gene model could be more accurate to predict patient survival.

\section{Pathway analysis of collagen family genes}

GSEA results showed that most of the gene sets were up-regulated in the high-risk group, and the top twenty enriched gene sets were given in Tables S3-S8. The gene sets that were closely associated with tumorigenesis were shown in Fig. 4. For instance, gene sets of PDGF, RB/P107, AKT/MTOR and p53 were significantly up-regulated according to Oncogenic Signatures Gene Sets (Figs. 4A-4F). Based on Hallmark Gene Sets, the enriched gene sets included p53 pathway, oxidative phosphorylation, apoptosis, mitotic spindle, G2/M checkpoint and notch signaling (Figs. 4G-4L). Using KEGG Gene Sets as reference, the high-risk group was tightly correlated with oxidative phosphorylation, renal cell carcinoma, bladder cancer, small cell lung cancer, adherens junction and cell cycle (Figs. 4M-4R).

\section{Co-expression network analysis}

WCGNA was performed to find out the genes that were co-expressed with collagen family genes in ESCC tissues. Risk-score-based DEGs that were differentially expressed between high- and low-risk groups were determined and presented by the volcano plot (Fig. S3). The co-expression network of collagen genes and the risk-score-based DEGs were given in several modules (Fig. 5). Collagen family genes were displayed as red nodes, and the genes included in the 7-gene prediction model in TCGA were marked as bigger red nodes. The blue nodes represented the co-expressed genes. Another network was drawn to show the association between collagen family genes and seven representative enriched gene sets (PDGF, RB/p107, PI3K/Akt/mTOR pathway, p53 pathway, oxidative phosphorylation, apoptosis and cell cycle) from the GSEA results (Fig. S4). The red nodes were the collagen family genes with close connections to those gene sets. A big blue circle represented a gene set and the blue nodes were genes included in each set. Genes closer 
261

262

263

264

265

266

267

268

269

270

271

272

273

274

275

276

277

278

279

280

281

282

283

284

285

286

287

288

289

290

291

292

293

294

295

296

297

298

299

300

to the center were more tightly associated with the collagen genes.

\section{Discussion}

Although extensive research efforts have been focused on this field in past decades, efficient detection methods for early ESCC and accurate prediction against complicated ESCC patients still remain an open issue. Recently, studies have found that the expression of certain genes, such as MCT4, ZNF750, Gli1, etc. was highly related to the occurrence and development of ESCC, and they might be applied as ideal biomarkers for ESCC (Cheng et al. 2018; Li et al. 2018; Nambara et al. 2017; Yang et al. 2017; Zhang et al. 2018b). In addition, the aberrant expression of a few collagen family genes has also been reported to be significantly associated with the prognosis of ESCC patients. However, most works only focused on single or limited genes, and the predictive ability was barely satisfactory. Herein, we provided a more systematic analysis of the whole collagen family gene expression to evaluate the potential roles and clinical significance of collagen genes in ESCC.

We found that most of the collagen genes were up-regulated in ESCC tissues when compared to normal controls, half of which were identified as DEGs (Figs. 1A and 1B). Among them, the expression of COL1A1, COL10A1 and COL11A1 was particularly higher, and that of COL4A4, COL6A5 and COL14A1 was especially lower in tumor tissues, indicating their possible roles as diagnostic markers for ESCC. Consistently, several studies have shown that COL1A1, COL10A1 and COL11A1 were notably overexpressed in ESCC compared to normal tissues (Fang et al. 2019; He et al. 2017; Karagoz et al. 2016; Senthebane et al. 2018; Zhang et al. 2018a). Also, COL4A4 was also found to be down-regulated in esophageal tumor tissues (Chattopadhyay et al. 2009). Additionally, among the DEGs, COL7A1 was observed to be up-regulated in ESCC tissues (Kita et al. 2009). In our works, COL6A5, COL14A1 and some other collagen genes were reported to be significantly up- or down-regulated in ESCC tissues for the first time.

In the survival analysis, COL6A5 and COL18A1 were validated to be significantly related to overall survival of ESCC patients. Previous studies demonstrated that the COL6A5 expression was significantly associated with depressed behavior and atopic dermatitis (Soderhall et al. 2007; Zhan et al. 2017), but no articles manifested its correlation with cancer. In addition, COL18A1 has been proved to be a promising biomarker for ovarian cancer and was possibly involved in the progression of bladder cancer (Fang et al. 2013; Peters et al. 2005). In this study, ESCC patients with low COL6A5 expression or high COL18A1 expression showed poor overall survival (Figs. $2 \mathrm{C}-2 \mathrm{~F}$ ), implying the expression of COL6A5 or COL18A1 as a potential indicator for the prognosis of ESCC patients. Moreover, the variations that affect the expression of COL6A5 and COL18A1 possibly have effects on the progression of ESCC. Activating COL6A5 or inhibiting COL18A1 might improve the therapeutic efficiency and the life-span of ESCC patients.

Because the expression of one gene is usually influenced by various factors, ideal effect may not be attained by using a single gene as a predictor. Indeed, COL6A5 achieved an AUC value over 0.60 only in TCGA (Fig. S1), making the requirement of another more powerful prediction method. Based on the selected collagen DEGs ( 7 genes in TCGA and 9 genes in GEO, both 
301

302

303

304

305

306

307

308

309

310

311

312

313

314

315

316

317

318

319

320

321

322

323

324

325

326

327

328

329

330

331

332

333

334

335

336

337

338

339

340

341

including COL6A5), we established two new prediction models. Importantly, such DEGs-based models exhibited better predictive ability than conventional prognostic models according to TNM staging. The 7-gene model in TCGA had especially higher predictive accuracy of $86 \%$. One possible reason was that the RNA sequencing technology applied to TCGA was more accurate than the gene chip technology used in GEO. In summary, this 7-gene prediction model is greatly promising to predict the prognosis of ESCC patients and help determine next therapeutic regimens.

Furthermore, GSEA was used to identify significantly enriched gene sets and potentially relevant pathways (Fig. 4). The results showed that based on Oncogenic Signatures Gene Sets, gene sets of PDGF, RB/P107 and AKT/MTOR were significantly enriched in the high-risk group. It has been reported that PDGF receptor-beta increased the expression of COL1A2 through Akt/mTORC1 signaling pathway (Das et al. 2017). According to Oncogenic Signatures Gene Sets and Hallmark Gene Sets, the high-risk group was significantly related to p53 and p53 pathway, which suggested that collagen genes might be highly associated with the p53 or its related pathway in ESCC. Earlier studies proved that enhanced expression of ectopic p53 in dermal fibroblasts inhibited basal and TGF-beta-stimulated collagen gene expression, and the absence of cellular p53 was correlated with increased transcriptional activity of the Type I collagen gene (COL1A2) and collagen synthesis (Ghosh et al. 2004). Moreover, the type IV collagen expression was inversely related to p53 in malignant tumors (Bar et al. 2004). Oxidative phosphorylation related genes were found to be up-regulated in the high-risk group by both Hallmark Gene Sets and KEGG Gene Sets. Indeed, some reports demonstrated that oxidative phosphorylation signature occurred when collagen density was decreased, and the change of collagen density microenvironment regulated the metabolism of cancer cells (Mah et al. 2018; Morris et al. 2016). As for apoptosis, an earlier study has shown that Type IV collagen could stimulate cancer cell proliferation, migration, and inhibit apoptosis (Ohlund et al. 2013). Additionally, the gene sets of mitotic spindle, G2/M checkpoint and cell cycle were enriched in the high-risk group as well, implying that collagen might regulate the cell cycle of ESCC cells. Furthermore, it was indicated that the high-risk group was markedly associated with renal cell carcinoma, bladder cancer and small cell lung cancer. These results were consistent with previous studies that collagen gene expression was correlated with the poor prognosis of those cancers (Koskimaki et al. 2010; Wan et al. 2015; Xu et al. 2017; Zeng et al. 2018).

As shown by the co-expression network (Fig. 5), a few collagen family genes such as COL1A1, COL11A1, COL6A6, and COL19A1, were co-expressed with NETO1, NEUROD2, and NRG3, which are the genes involved in neural functions. These findings could be verified by earlier articles to some extent (McCarthy \& Hay 1991; Perris et al. 1993a; Perris et al. 1993b). COL11A1 was also observed to be co-expressed with tumor suppressor candidate 7 (TUSC7), further validating the possible role of COL11A1 in the occurrence of ESCC. Beyond that, some potassium channel related genes (KCNA2, KCNE1B, KCNH1, KCNJ4, and KCNK4) were co-expressed with collagen genes in a way, revealing that collagen genes might be correlated with the regulation of potassium channels in ESCC. As for the two potential prognostic biomarkers, COL18A1 only showed close relations with collagen family members, while COL6A5 was associated with two other genes in this network, ROBO2 and MIR548A3. ROBO2 has been identified as a candidate 
342

343

tumor suppressor (Trifonov et al. 2013), and the alteration of its expression might play a role in malignant tumors of digestive tract including gastric and colorectal cancers (Je et al. 2013).

Apart from what is aforementioned, there are still some limitations of this research. For instance, the prediction model was comprised of several genes, making it difficult to conduct cellular experiments by targeting a single gene to confirm its predictive effect. Aside from it, the characteristics of patient samples, as well as the methodology utilized in TCGA, were somewhat different from that in GEO, which may explain the different results coming from the two datasets. For example, TCGA uses the RNA sequence technology while GEO applies the gene chip technology to detect gene expression of patient tissues. Besides, TCGA mainly collected data from white people, whereas the majority of patients in GEO (GSE53625) were Asian. Therefore, there was no a single gene that exhibited significant $P$ values in both datasets in the survival analysis, and the selected genes driving the prediction model in one dataset were not completely identical to those in another dataset. Further validation of these outcomes requires more clinical information and biological experiments in the future.

\section{Conclusions}

In summary, this study identified 22 collagen family genes that were significantly expressed higher or lower in ESCC compared to normal tissues. Among them, COL1A1, COL10A1, COL11A1, COL4A4, COL6A5 and COL14A1 were the most distinct ones and possessed the potential in ESCC diagnosis. Besides, COL6A5 and COL18A1 showed strong correlations with overall survival of ESCC patients and might be robust prognostic biomarkers for ESCC. Furthermore, we established a 7-gene prediction model with high performance to predict the prognosis of ESCC patients. In terms of the underlying mechanism, collagen genes might be associated with $\mathrm{PI3K} / \mathrm{Akt} / \mathrm{mTOR}$ pathway, p53 pathway, oxidative phosphorylation, apoptosis and cell cycle during the progression of ESCC. Our works may further benefit the diagnosis, prognosis and treatments for ESCC patients.

\section{References}

Bar JK, Grelewski P, Popiela A, Noga L, and Rabczynski J. 2004. Type IV collagen and CD44v6 expression in benign, malignant primary and metastatic ovarian tumors: correlation with Ki-67 and p53 immunoreactivity. Gynecol Oncol 95:23-31. 10.1016/j.ygyno.2004.06.046

Beer DG, Kardia SL, Huang CC, Giordano TJ, Levin AM, Misek DE, Lin L, Chen G, Gharib TG, Thomas DG, Lizyness ML, Kuick R, Hayasaka S, Taylor JM, Iannettoni MD, Orringer MB, and Hanash S. 2002. Gene-expression profiles predict survival of patients with lung adenocarcinoma. Nature Medicine 8:816-824. 10.1038/nm733

Bray F, Ferlay J, Soerjomataram I, Siegel RL, Torre LA, and Jemal A. 2018. Global cancer statistics 2018: GLOBOCAN estimates of incidence and mortality worldwide for 36 cancers in 185 countries. $C A$ Cancer J Clin. 10.3322/caac. 21492

Chai R, Zhang K, Wang K, Li G, Huang R, Zhao Z, Liu Y, and Chen J. 2018. A novel gene signature based on 
381

382

383

384

385

386

387

388

389

390

391

392

393

394

395

396

397

398

399

400

401

402

403

404

405

406

407

408

409

410

411

412

413

414

415

416

417

418

419

420

421 five glioblastoma stem-like cell relevant genes predicts the survival of primary glioblastoma. $J$ Cancer Res Clin Oncol 144:439-447. 10.1007/s00432-017-2572-6

Chattopadhyay I, Phukan R, Singh A, Vasudevan M, Purkayastha J, Hewitt S, Kataki A, Mahanta J, Kapur S, and Saxena S. 2009. Molecular profiling to identify molecular mechanism in esophageal cancer with familial clustering. Oncol Rep 21:1135-1146.

Cheng B, Chen X, Li Y, Huang X, and Yu J. 2018. Prognostic value of monocarboxylate transporter 4 in patients with esophageal squamous cell carcinoma. Oncol Rep 40:2906-2915. 10.3892/or.2018.6706

Codipilly DC, Qin Y, Dawsey SM, Kisiel J, Topazian M, Ahlquist D, and Iyer PG. 2018. Screening for esophageal squamous cell carcinoma: recent advances. Gastrointest Endosc 88:413-426. 10.1016/j.gie.2018.04.2352

D'Journo XB. 2018. Clinical implication of the innovations of the 8(th) edition of the TNM classification for esophageal and esophago-gastric cancer. $J$ Thorac Dis 10:S2671-S2681. 10.21037/jtd.2018.03.182

Das F, Ghosh-Choudhury N, Venkatesan B, Kasinath BS, and Ghosh Choudhury G. 2017. PDGF receptor-beta uses Akt/mTORC1 signaling node to promote high glucose-induced renal proximal tubular cell collagen I (alpha2) expression. Am J Physiol Renal Physiol 313:F291-F307. 10.1152/ajprenal.00666.2016

Fan NJ, Gao CF, Wang CS, Zhao G, Lv JJ, Wang XL, Chu GH, Yin J, Li DH, Chen X, Yuan XT, and Meng NL. 2012. Identification of the up-regulation of TP-alpha, collagen alpha-1(VI) chain, and S100A9 in esophageal squamous cell carcinoma by a proteomic method. J Proteomics 75:3977-3986. 10.1016/j.jprot.2012.05.008

Fang S, Dai Y, Mei Y, Yang M, Hu L, Yang H, Guan X, and Li J. 2019. Clinical significance and biological role of cancer-derived Type I collagen in lung and esophageal cancers. Thorac Cancer. 10.1111/17597714.12947

Fang ZQ, Zang WD, Chen R, Ye BW, Wang XW, Yi SH, Chen W, He F, and Ye G. 2013. Gene expression profile and enrichment pathways in different stages of bladder cancer. Genet Mol Res 12:1479-1489. 10.4238/2013.May.6.1

Ghosh AK, Bhattacharyya S, and Varga J. 2004. The tumor suppressor p53 abrogates Smad-dependent collagen gene induction in mesenchymal cells. J Biol Chem 279:47455-47463. 10.1074/jbc.M403477200

Giussani M, Landoni E, Merlino G, Turdo F, Veneroni S, Paolini B, Cappelletti V, Miceli R, Orlandi R, Triulzi T, and Tagliabue E. 2018. Extracellular matrix proteins as diagnostic markers of breast carcinoma. $J$ Cell Physiol 233:6280-6290. 10.1002/jcp.26513

Guo Q, Zheng M, Xu Y, Wang N, and Zhao W. 2018. miR-384 induces apoptosis and autophagy of non-small cell lung cancer(NSCLC) cells through the negative regulation of Collagen alpha-1(X) chain(COL10A1) gene. Biosci Rep. 10.1042/BSR20181523

He Y, Liu J, Zhao Z, and Zhao H. 2017. Bioinformatics analysis of gene expression profiles of esophageal squamous cell carcinoma. Dis Esophagus 30:1-8. 10.1093/dote/dow018

Je EM, Gwak M, Oh H, Choi MR, Choi YJ, Lee SH, and Yoo NJ. 2013. Frameshift mutations of axon guidance genes ROBO1 and ROBO2 in gastric and colorectal cancers with microsatellite instability. Pathology 45:645-650. 10.1097/PAT.0000000000000007

Karagoz K, Lehman HL, Stairs DB, Sinha R, and Arga KY. 2016. Proteomic and Metabolic Signatures of Esophageal Squamous Cell Carcinoma. Curr Cancer Drug Targets.

Kita Y, Mimori K, Tanaka F, Matsumoto T, Haraguchi N, Ishikawa K, Matsuzaki S, Fukuyoshi Y, Inoue H, 
Natsugoe S, Aikou T, and Mori M. 2009. Clinical significance of LAMB3 and COL7A1 mRNA in esophageal squamous cell carcinoma. Eur J Surg Oncol 35:52-58. 10.1016/j.ejso.2008.01.025

Koskimaki JE, Karagiannis ED, Tang BC, Hammers H, Watkins DN, Pili R, and Popel AS. 2010. Pentastatin1 , a collagen IV derived 20-mer peptide, suppresses tumor growth in a small cell lung cancer xenograft model. BMC Cancer 10:29. 10.1186/1471-2407-10-29

Kosugi S, Nishimaki T, Kanda T, Nakagawa S, Ohashi M, and Hatakeyama K. 2004. Clinical significance of serum carcinoembryonic antigen, carbohydrate antigen 19-9, and squamous cell carcinoma antigen levels in esophageal cancer patients. World J Surg 28:680-685. 10.1007/s00268-004-6865-y

Langfelder P, and Horvath S. 2008. WGCNA: an R package for weighted correlation network analysis. $B M C$ Bioinformatics 9:559. 10.1186/1471-2105-9-559

Lao-Sirieix P, and Fitzgerald RC. 2012. Screening for oesophageal cancer. Nat Rev Clin Oncol 9:278-287. 10.1038/nrclinonc.2012.35

Law CW, Alhamdoosh M, Su S, Dong X, Tian L, Smyth GK, and Ritchie ME. 2016. RNA-seq analysis is easy as 1-2-3 with limma, Glimma and edgeR. F1000Res 5. 10.12688/f1000research.9005.3

Li J, Chen Z, Tian L, Zhou C, He MY, Gao Y, Wang S, Zhou F, Shi S, Feng X, Sun N, Liu Z, Skogerboe G, Dong J, Yao R, Zhao Y, Sun J, Zhang B, Yu Y, Shi X, Luo M, Shao K, Li N, Qiu B, Tan F, Chen R, and He J. 2014. LncRNA profile study reveals a three-lncRNA signature associated with the survival of patients with oesophageal squamous cell carcinoma. Gut 63:1700-1710. 10.1136/gutjnl-2013-305806

Li K, Liu Y, Xu S, and Wang J. 2018. PPM1D Functions as Oncogene and is Associated with Poor Prognosis in Esophageal Squamous Cell Carcinoma. Pathol Oncol Res. 10.1007/s12253-018-0518-1

Liu W, Li L, Ye H, Tao H, and He H. 2018. Role of COL6A3 in colorectal cancer. Oncol Rep 39:2527-2536. 10.3892/or.2018.6331

Lossos IS, Czerwinski DK, Alizadeh AA, Wechser MA, Tibshirani R, Botstein D, and Levy R. 2004. Prediction of survival in diffuse large-B-cell lymphoma based on the expression of six genes. $N$ Engl J Med 350:1828-1837. 10.1056/NEJMoa032520

Ludemann L, Grieger W, Wurm R, Wust P, and Zimmer C. 2006. Glioma assessment using quantitative blood volume maps generated by T1-weighted dynamic contrast-enhanced magnetic resonance imaging: a receiver operating characteristic study. Acta Radiol 47:303-310.

Mah EJ, Lefebvre A, McGahey GE, Yee AF, and Digman MA. 2018. Collagen density modulates triple-negative breast cancer cell metabolism through adhesion-mediated contractility. Sci Rep 8:17094. 10.1038/s41598-018-35381-9

McCarthy RA, and Hay ED. 1991. Collagen I, laminin, and tenascin: ultrastructure and correlation with avian neural crest formation. Int J Dev Biol 35:437-452.

Metz CE. 1978. Basic principles of ROC analysis. Semin Nucl Med 8:283-298.

Morris BA, Burkel B, Ponik SM, Fan J, Condeelis JS, Aguirre-Ghiso JA, Castracane J, Denu JM, and Keely PJ. 2016. Collagen Matrix Density Drives the Metabolic Shift in Breast Cancer Cells. EBioMedicine 13:146-156. 10.1016/j.ebiom.2016.10.012

Nambara S, Masuda T, Tobo T, Kidogami S, Komatsu H, Sugimachi K, Saeki H, Oki E, Maehara Y, and Mimori K. 2017. Clinical significance of ZNF750 gene expression, a novel tumor suppressor gene, in esophageal squamous cell carcinoma. Oncol Lett 14:1795-1801. 10.3892/ol.2017.6341

Obuchowski NA. 2003. Receiver operating characteristic curves and their use in radiology. Radiology 229:3-8. 
10.1148/radiol.2291010898

Ohlund D, Franklin O, Lundberg E, Lundin C, and Sund M. 2013. Type IV collagen stimulates pancreatic cancer cell proliferation, migration, and inhibits apoptosis through an autocrine loop. BMC Cancer 13:154. 10.1186/1471-2407-13-154

Pennathur A, Gibson MK, Jobe BA, and Luketich JD. 2013. Oesophageal carcinoma. Lancet 381:400-412. 10.1016/S0140-6736(12)60643-6

Perris R, Kuo HJ, Glanville RW, and Bronner-Fraser M. 1993a. Collagen type VI in neural crest development: distribution in situ and interaction with cells in vitro. Dev Dyn 198:135-149. 10.1002/aja.1001980207

Perris R, Kuo HJ, Glanville RW, Leibold S, and Bronner-Fraser M. 1993b. Neural crest cell interaction with type VI collagen is mediated by multiple cooperative binding sites within triple-helix and globular domains. Exp Cell Res 209:103-117. 10.1006/excr.1993.1290

Peters DG, Kudla DM, Deloia JA, Chu TJ, Fairfull L, Edwards RP, and Ferrell RE. 2005. Comparative gene expression analysis of ovarian carcinoma and normal ovarian epithelium by serial analysis of gene expression. Cancer Epidemiol Biomarkers Prev 14:1717-1723. 10.1158/1055-9965.EPI-04-0704

Phipson B, Lee S, Majewski IJ, Alexander WS, and Smyth GK. 2016. Robust Hyperparameter Estimation Protects against Hypervariable Genes and Improves Power to Detect Differential Expression. Ann Appl Stat 10:946-963. 10.1214/16-AOAS920

Ricard-Blum S. 2011. The collagen family. Cold Spring Harb Perspect Biol 3:a004978. 10.1101/cshperspect.a004978

Ritchie ME, Phipson B, Wu D, Hu Y, Law CW, Shi W, and Smyth GK. 2015. limma powers differential expression analyses for RNA-sequencing and microarray studies. Nucleic Acids Res 43:e47. 10.1093/nar/gkv007

Robinson MD, McCarthy DJ, and Smyth GK. 2010. edgeR: a Bioconductor package for differential expression analysis of digital gene expression data. Bioinformatics 26:139-140. 10.1093/bioinformatics/btp616

Rong L, Huang W, Tian S, Chi X, Zhao P, and Liu F. 2018. COL1A2 is a Novel Biomarker to Improve Clinical Prediction in Human Gastric Cancer: Integrating Bioinformatics and Meta-Analysis. Pathol Oncol Res 24:129-134. 10.1007/s12253-017-0223-5

Rossi F, MacLean HE, Yuan W, Francis RO, Semenova E, Lin CS, Kronenberg HM, and Cobrinik D. 2002. p107 and p130 Coordinately regulate proliferation, Cbfa1 expression, and hypertrophic differentiation during endochondral bone development. Dev Biol 247:271-285.

Senthebane DA, Jonker T, Rowe A, Thomford NE, Munro D, Dandara C, Wonkam A, Govender D, Calder B, Soares NC, Blackburn JM, Parker MI, and Dzobo K. 2018. The Role of Tumor Microenvironment in Chemoresistance: 3D Extracellular Matrices as Accomplices. Int J Mol Sci 19. 10.3390/ijms19102861

Shen L, Yang M, Lin Q, Zhang Z, Zhu B, and Miao C. 2016. COL11A1 is overexpressed in recurrent non-small cell lung cancer and promotes cell proliferation, migration, invasion and drug resistance. Oncol Rep 36:877-885. 10.3892/or.2016.4869

Smyth GK, and Speed T. 2003. Normalization of cDNA microarray data. Methods 31:265-273.

Soderhall C, Marenholz I, Kerscher T, Ruschendorf F, Esparza-Gordillo J, Worm M, Gruber C, Mayr G, Albrecht M, Rohde K, Schulz H, Wahn U, Hubner N, and Lee YA. 2007. Variants in a novel epidermal collagen gene (COL29A1) are associated with atopic dermatitis. PLoS Biol 5:e242. 10.1371/journal.pbio.0050242 
504

505

506

507

508

509

510

511

512

513

514

515

516

517

518

519

520

521

522

523

524

525

526

527

528

529

530

531

532

533

534

535

536

537

538

539

540

541

542

543

544

Sorushanova A, Delgado LM, Wu Z, Shologu N, Kshirsagar A, Raghunath R, Mullen AM, Bayon Y, Pandit A, Raghunath M, and Zeugolis DI. 2018. The Collagen Suprafamily: From Biosynthesis to Advanced Biomaterial Development. Adv Mater:e1801651. 10.1002/adma.201801651

Spruance SL, Reid JE, Grace M, and Samore M. 2004. Hazard ratio in clinical trials. Antimicrob Agents Chemother 48:2787-2792. 10.1128/AAC.48.8.2787-2792.2004

Subramanian A, Tamayo P, Mootha VK, Mukherjee S, Ebert BL, Gillette MA, Paulovich A, Pomeroy SL, Golub TR, Lander ES, and Mesirov JP. 2005. Gene set enrichment analysis: a knowledge-based approach for interpreting genome-wide expression profiles. Proc Natl Acad Sci U S A 102:15545-15550. 10.1073/pnas.0506580102

Trifonov V, Pasqualucci L, Dalla Favera R, and Rabadan R. 2013. MutComFocal: an integrative approach to identifying recurrent and focal genomic alterations in tumor samples. BMC Syst Biol 7:25. $10.1186 / 1752-0509-7-25$

Wan F, Wang H, Shen Y, Zhang H, Shi G, Zhu Y, Dai B, and Ye D. 2015. Upregulation of COL6A1 is predictive of poor prognosis in clear cell renal cell carcinoma patients. Oncotarget 6:27378-27387. 10.18632/oncotarget.4860

Wang GQ, Jiao GG, Chang FB, Fang WH, Song JX, Lu N, Lin DM, Xie YQ, and Yang L. 2004. Long-term results of operation for 420 patients with early squamous cell esophageal carcinoma discovered by screening. Ann Thorac Surg 77:1740-1744. 10.1016/j.athoracsur.2003.10.098

Wang Z, Song Q, Yang Z, Chen J, Shang J, and Ju W. 2018. Construction of immune-related risk signature for renal papillary cell carcinoma. Cancer Med. 10.1002/cam4.1905

Xu F, Chang K, Ma J, Qu Y, Xie H, Dai B, Gan H, Zhang H, Shi G, Zhu Y, Zhu Y, Shen Y, and Ye D. 2017. The Oncogenic Role of COL23A1 in Clear Cell Renal Cell Carcinoma. Sci Rep 7:9846. 10.1038/s41598-017-10134-2

Yang Z, Cui Y, Ni W, Kim S, and Xuan Y. 2017. Gli1, a potential regulator of esophageal cancer stem cell, is identified as an independent adverse prognostic factor in esophageal squamous cell carcinoma. $J$ Cancer Res Clin Oncol 143:243-254. 10.1007/s00432-016-2273-6

Zeng XT, Liu XP, Liu TZ, and Wang XH. 2018. The clinical significance of COL5A2 in patients with bladder cancer: A retrospective analysis of bladder cancer gene expression data. Medicine (Baltimore) 97:e0091. 10.1097/MD.0000000000010091

Zhan H, Huang F, Yan F, Zhao Z, Zhang J, Cui T, Yang F, Hai G, Jia X, and Shi Y. 2017. Alterations in splenic function and gene expression in mice with depressive-like behavior induced by exposure to corticosterone. Int J Mol Med 39:327-336. 10.3892/ijmm.2017.2850

Zhang B, Zhang C, Yang X, Chen Y, Zhang H, Liu J, and Wu Q. 2018a. Cytoplasmic collagen XIalphaI as a prognostic biomarker in esophageal squamous cell carcinoma. Cancer Biol Ther 19:364-372. 10.1080/15384047.2018.1423915

Zhang Y, Xu Y, Li Z, Zhu Y, Wen S, Wang M, Lv H, Zhang F, and Tian Z. 2018b. Identification of the key transcription factors in esophageal squamous cell carcinoma. $J$ Thorac Dis 10:148-161. 10.21037/jtd.2017.12.27

Zhang Z, Fang C, Wang Y, Zhang J, Yu J, Zhang Y, Wang X, and Zhong J. 2018c. COL1A1: A potential therapeutic target for colorectal cancer expressing wild-type or mutant KRAS. Int J Oncol 53:18691880. 10.3892/ijo.2018.4536 
545 Zhang ZL, Zhao LJ, Chai L, Zhou SH, Wang F, Wei Y, Xu YP, and Zhao P. 2017. Seven LncRNA-mRNA

546 based risk score predicts the survival of head and neck squamous cell carcinoma. Sci Rep 7:309.

$547 \quad 10.1038 / \mathrm{s} 41598-017-00252-2$

548 Zhao S, Cai J, Li J, Bao G, Li D, Li Y, Zhai X, Jiang C, and Fan L. 2017. Bioinformatic Profiling Identifies a

549 Glucose-Related Risk Signature for the Malignancy of Glioma and the Survival of Patients. Mol

550 Neurobiol 54:8203-8210. 10.1007/s12035-016-0314-4

551 


\section{Table $\mathbf{1}$ (on next page)}

Univariate and multivariate analyses of clinicopathological characteristics for overall survival in ESCC patients from the TCGA dataset $(N=95)$.

Characteristics with $P<0.3$ in the univariate analysis were further screened in the multivariate analysis. $\mathrm{HR}$, hazard ratio; $\mathrm{Cl}$, confidence interval; TNM stage, tumor-nodemetastasis stage; T stage, stage of tumor invasion; $\mathrm{N}$ stage, stage of regional lymph node invasion. 


\begin{tabular}{|c|c|c|c|c|c|}
\hline \multirow{2}{*}{ Variables } & \multirow{2}{*}{$\mathrm{n}(\%)$} & \multicolumn{2}{|c|}{ Univariate analysis } & \multicolumn{2}{|c|}{ Multivariate analysis } \\
\hline & & HR $(95 \%$ CI $)$ & $P$ & HR $(95 \%$ CI $)$ & $P$ \\
\hline \multicolumn{6}{|l|}{ Age } \\
\hline$<60$ & $56(58.9 \%)$ & 1 (Reference) & & & \\
\hline$\geq 60$ & $39(41.1 \%)$ & $1.296(0.631-2.662)$ & 0.461 & & \\
\hline \multicolumn{6}{|l|}{ Sex } \\
\hline Male & $80(84.2 \%)$ & 1 (Reference) & & 1 (Reference) & \\
\hline Female & $15(15.8 \%)$ & $0.175(0.041-0.756)$ & 0.020 & $0.206(0.043-0.978)$ & 0.047 \\
\hline \multicolumn{6}{|l|}{ TNM Stage } \\
\hline $\mathrm{I}+\mathrm{II}$ & $63(66.3 \%)$ & 1 (Reference) & & 1 (Reference) & \\
\hline $\mathrm{III}+\mathrm{IV}$ & $31(32.6 \%)$ & $2.443(1.191-5.011)$ & 0.015 & $0.921(0.321-2.643)$ & 0.879 \\
\hline Missing & $1(1.1 \%)$ & & & & \\
\hline \multicolumn{6}{|l|}{ T Stage } \\
\hline $\mathrm{T} 1+\mathrm{T} 2$ & $40(42.1 \%)$ & 1 (Reference) & & & \\
\hline $\mathrm{T} 3+\mathrm{T} 4$ & $54(56.8 \%)$ & $1.351(0.649-2.811)$ & 0.422 & & \\
\hline Missing & $1(1.1 \%)$ & & & & \\
\hline \multicolumn{6}{|l|}{ Tumor Grade } \\
\hline $\mathrm{G} 1+\mathrm{G} 2$ & $65(68.4 \%)$ & 1 (Reference) & & & \\
\hline G3 & $21(22.1 \%)$ & $0.736(0.277-1.950)$ & 0.537 & & \\
\hline Missing & $9(9.5 \%)$ & & & & \\
\hline \multicolumn{6}{|l|}{ N Stage } \\
\hline $\mathrm{N} 0+\mathrm{N} 1$ & $84(88.4 \%)$ & 1 (Reference) & & 1 (Reference) & \\
\hline $\mathrm{N} 2+\mathrm{N} 3$ & $9(9.5 \%)$ & $3.265(1.302-8.189)$ & 0.012 & $6.738(1.493-30.399)$ & 0.013 \\
\hline Missing & $2(2.1 \%)$ & & & & \\
\hline \multicolumn{6}{|l|}{ Tumor Location } \\
\hline Upper+Middle & $50(52.6 \%)$ & 1 (Reference) & & & \\
\hline Lower & $44(46.3 \%)$ & $0.958(0.448-2.051)$ & 0.913 & & \\
\hline Missing & $1(1.1 \%)$ & & & & \\
\hline \multicolumn{6}{|l|}{ Alcohol Use } \\
\hline No & $25(26.3 \%)$ & 1 (Reference) & & 1 (Reference) & \\
\hline Yes & $68(71.6 \%)$ & $2.172(0.751-6.276)$ & 0.152 & $4.755(1.054-21.457)$ & 0.043 \\
\hline Missing & $2(2.1 \%)$ & & & & \\
\hline \multicolumn{6}{|l|}{ Tobacco use } \\
\hline No & $44(46.3 \%)$ & 1 (Reference) & & 1 (Reference) & \\
\hline Yes & $51(53.7 \%)$ & $1.965(0.901-4.285)$ & 0.089 & $1.095(0.440-2.725)$ & 0.845 \\
\hline \multicolumn{6}{|l|}{ Race } \\
\hline Asian & $45(47.4 \%)$ & 1 (Reference) & & 1 (Reference) & \\
\hline White+Other & $47(49.5 \%)$ & $1.570(0.688-3.581)$ & 0.284 & $2.021(0.782-5.223)$ & 0.146 \\
\hline Missing & $3(3.2 \%)$ & & & & \\
\hline
\end{tabular}




\section{Table 2 (on next page)}

Univariate and multivariate analyses of clinicopathological characteristics for overall survival in ESCC patients from the GEO dataset $(N=179)$.

Characteristics with $P<0.3$ in the univariate analysis were further screened in the multivariate analysis. $\mathrm{HR}$, hazard ratio; $\mathrm{Cl}$, confidence interval; TNM stage, tumor-nodemetastasis stage; $\mathrm{T}$ stage, stage of tumor invasion; $\mathrm{N}$ stage, stage of regional lymph node invasion. 


\begin{tabular}{|c|c|c|c|c|c|}
\hline \multirow{2}{*}{ Variables } & \multirow{2}{*}{$\mathrm{n}(\%)$} & \multicolumn{2}{|c|}{ Univariate analysis } & \multicolumn{2}{|c|}{ Multivariate analysis } \\
\hline & & HR $(95 \%$ CI $)$ & $P$ & HR $(95 \%$ CI $)$ & $P$ \\
\hline \multicolumn{6}{|l|}{ Age } \\
\hline$<60$ & $91(50.8 \%)$ & 1 (Reference) & & 1 (Reference) & \\
\hline$\geq 60$ & $88(49.2 \%)$ & $1.574(1.072-2.311)$ & 0.021 & $1.451(0.980-2.147)$ & 0.063 \\
\hline \multicolumn{6}{|l|}{ Sex } \\
\hline Male & $146(81.6 \%)$ & 1 (Reference) & & & \\
\hline Female & $33(18.4 \%)$ & $1.277(0.798-2.044)$ & 0.307 & & \\
\hline \multicolumn{6}{|l|}{ TNM Stage } \\
\hline $\mathrm{I}+\mathrm{II}$ & $87(48.6 \%)$ & 1 (Reference) & & 1 (Reference) & \\
\hline $\mathrm{III}+\mathrm{IV}$ & $92(51.4 \%)$ & $2.155(1.448-3.207)$ & $<0.001$ & $2.066(1.322-3.228)$ & 0.001 \\
\hline \multicolumn{6}{|l|}{ T Stage } \\
\hline $\mathrm{T} 1+\mathrm{T} 2$ & $39(21.8 \%)$ & 1 (Reference) & & & \\
\hline $\mathrm{T} 3+\mathrm{T} 4$ & $140(78.2 \%)$ & $1.091(0.687-1.732)$ & 0.712 & & \\
\hline \multicolumn{6}{|l|}{ Tumor Grade } \\
\hline $\mathrm{G} 1+\mathrm{G} 2$ & $99(55.3 \%)$ & 1 (Reference) & & 1 (Reference) & \\
\hline G3 & $80(44.7 \%)$ & $1.391(0.951-2.037)$ & 0.089 & $1.269(0.860-1.873)$ & 0.230 \\
\hline \multicolumn{6}{|l|}{ N Stage } \\
\hline $\mathrm{N} 0+\mathrm{N} 1$ & $145(81.0 \%)$ & 1 (Reference) & & 1 (Reference) & \\
\hline $\mathrm{N} 2+\mathrm{N} 3$ & $34(19.0 \%)$ & $1.644(1.048-2.577)$ & 0.030 & $1.062(0.644-1.751)$ & 0.814 \\
\hline \multicolumn{6}{|l|}{ Tumor Location } \\
\hline Upper+Middle & $117(65.4 \%)$ & 1 (Reference) & & & \\
\hline Lower & $62(34.6 \%)$ & $0.823(0.546-1.242)$ & 0.354 & & \\
\hline \multicolumn{6}{|l|}{ Alcohol Use } \\
\hline No & $73(40.8 \%)$ & 1 (Reference) & & & \\
\hline Yes & $106(59.2 \%)$ & $0.864(0.588-1.269)$ & 0.456 & & \\
\hline \multicolumn{6}{|l|}{ Tobacco Use } \\
\hline No & $65(36.3 \%)$ & 1 (Reference) & & 1 (Reference) & \\
\hline Yes & $114(63.7 \%)$ & $0.749(0.508-1.105)$ & 0.145 & $0.753(0.505-1.122)$ & 0.163 \\
\hline \multicolumn{6}{|l|}{ Pneumonia } \\
\hline No & $164(91.6 \%)$ & 1 (Reference) & & & \\
\hline Yes & $15(8.4 \%)$ & $1.425(0.719-2.824)$ & 0.310 & & \\
\hline
\end{tabular}

1 


\section{Table 3(on next page)}

Correlation of collagen family gene expression and clinicopathological characteristics of ESCC patients from the TCGA dataset.

Superscripts of the correlation coefficients represent $P$ values. * correlation with $P<0.05$; ** correlation with $P<0.01$. 


\begin{tabular}{|c|c|c|c|c|c|c|}
\hline Gene & $\mathrm{Ag} \geq 60$ & $\begin{array}{c}\text { Sex } \\
\text { (Female) }\end{array}$ & $\begin{array}{c}\text { TNM Stage } \\
\text { III/IV }\end{array}$ & $\begin{array}{c}\text { N stage } \\
(\mathrm{N} 1+\mathrm{N} 2)\end{array}$ & $\begin{array}{c}\text { Tumor Grade } \\
\text { (G3) }\end{array}$ & $\begin{array}{c}\text { Tumor Location } \\
\text { (Lower) }\end{array}$ \\
\hline COL1A1 & & $-0.222^{*} * 0.048$ & & & & \\
\hline COL1A2 & & $-0.222^{*} * 0.048$ & & & & \\
\hline \multicolumn{7}{|l|}{ COL2A1 } \\
\hline COL3A1 & & $-2.225^{*} 0.045$ & & & & \\
\hline \multicolumn{7}{|l|}{ COL4A1 } \\
\hline \multicolumn{7}{|l|}{ COL4A2 } \\
\hline \multicolumn{7}{|l|}{ COL4A3 } \\
\hline \multicolumn{7}{|l|}{ COL4A4 } \\
\hline \multicolumn{7}{|l|}{ COL4A5 } \\
\hline \multicolumn{7}{|l|}{ COL4A6 } \\
\hline \multicolumn{7}{|l|}{ COL5A1 } \\
\hline COL5A2 & & $-0.231^{* 0.039}$ & & & & \\
\hline COL5A3 & & $-0.229^{*} 0.041$ & & & & \\
\hline \multicolumn{7}{|l|}{ COL6A1 } \\
\hline \multicolumn{7}{|l|}{ COL6A2 } \\
\hline \multicolumn{7}{|l|}{ COL6A3 } \\
\hline \multicolumn{7}{|l|}{ COL6A5 } \\
\hline \multicolumn{7}{|l|}{ COL6A6 } \\
\hline COL7A1 & & & & & $-0.226^{* 0.046}$ & $-0.226^{* 0.046}$ \\
\hline \multicolumn{7}{|l|}{ COL8A1 } \\
\hline \multicolumn{7}{|l|}{ COL8A2 } \\
\hline \multicolumn{7}{|l|}{ COL9A1 } \\
\hline \multicolumn{7}{|l|}{ COL9A2 } \\
\hline COL9A3 & & $0.318^{* * 0.004}$ & & & & \\
\hline \multicolumn{7}{|l|}{ COL10A1 } \\
\hline \multicolumn{7}{|l|}{ COL11A1 } \\
\hline \multicolumn{7}{|l|}{ COL11A2 } \\
\hline COL12A1 & & & & & & $-0.288^{* 0.010}$ \\
\hline \multicolumn{7}{|l|}{ COL13A1 } \\
\hline \multicolumn{7}{|l|}{ COL14A1 } \\
\hline \multicolumn{7}{|l|}{ COL15A1 } \\
\hline COL16A1 & & & $-0.280^{*} 0.013$ & & $-0.280^{*} 0.013$ & \\
\hline COL17A1 & & & $-0.299^{* * 0} 0.008$ & & $-0.299^{* * 0.008}$ & \\
\hline \multicolumn{7}{|l|}{ COL18A1 } \\
\hline COL19A1 & & & & $0.367^{* *} 0.00$ & & \\
\hline \multicolumn{7}{|l|}{ COL20A1 } \\
\hline COL21A1 & & $0.243^{*} 0.030$ & & & & \\
\hline \multicolumn{7}{|l|}{ COL22A1 } \\
\hline COL23A1 & & & & & & \\
\hline
\end{tabular}


COL24A1

COL25A1

COL26A1

COL27A1 $-0.245^{*} 0.02$

COL28A1

1

Peer] reviewing PDF | (2019:03:35949:2:0:NEW 12 Aug 2019) 


\section{Table 4 (on next page)}

Correlation of collagen family gene expression and clinicopathological characteristics of ESCC patients in GEO.

Superscripts of the correlation coefficients represent $P$ values. * correlation with $P<0.05$; ** correlation with $P<0.01$. 


\begin{tabular}{|c|c|c|c|c|c|c|}
\hline Gene & Age $\geq 60$ & $\begin{array}{c}\text { Sex } \\
\text { (Female) }\end{array}$ & $\begin{array}{c}\text { TNM Stage } \\
\text { III+IV }\end{array}$ & $\begin{array}{c}\text { N stage } \\
(\mathrm{N} 1+\mathrm{N} 2)\end{array}$ & $\begin{array}{c}\text { Tumor Grade } \\
\text { (G3) }\end{array}$ & $\begin{array}{c}\text { Tumor Location } \\
\text { (Lower) }\end{array}$ \\
\hline \multicolumn{7}{|l|}{ COL1A1 } \\
\hline \multicolumn{7}{|l|}{ COL1A2 } \\
\hline \multicolumn{7}{|l|}{ COL2A1 } \\
\hline \multicolumn{7}{|l|}{ COL3A1 } \\
\hline \multicolumn{7}{|l|}{ COL4A1 } \\
\hline \multicolumn{7}{|l|}{ COL4A2 } \\
\hline COL4A3 & & & & & $0.149^{* 0.046}$ & $-0.162^{* 0.030}$ \\
\hline COL4A4 & & & & & & $-0.168^{* 0.024}$ \\
\hline \multicolumn{7}{|l|}{ COL4A5 } \\
\hline \multicolumn{7}{|l|}{ COL4A6 } \\
\hline \multicolumn{7}{|l|}{ COL5A1 } \\
\hline \multicolumn{7}{|l|}{ COL5A2 } \\
\hline COL5A3 & & & & & & $0.167^{* 0.026}$ \\
\hline \multicolumn{7}{|l|}{ COL6A1 } \\
\hline \multicolumn{7}{|l|}{ COL6A2 } \\
\hline \multicolumn{7}{|l|}{ COL6A3 } \\
\hline COL6A5 & & & & & $-0.173^{* 0.020}$ & \\
\hline \multicolumn{7}{|l|}{ COL6A6 } \\
\hline \multicolumn{7}{|l|}{ COL7A1 } \\
\hline COL8A1 & & $0.188^{* 0.012}$ & & & & \\
\hline \multicolumn{7}{|l|}{ COL8A2 } \\
\hline \multicolumn{7}{|l|}{ COL9A1 } \\
\hline COL9A2 & & & & $-0.175^{*} 0.019$ & & \\
\hline COL9A3 & & & & & $0.162^{*} 0.030$ & \\
\hline COL10A1 & & & & & $-0.151^{* 0.044}$ & \\
\hline \multicolumn{7}{|l|}{ COL11A1 } \\
\hline \multicolumn{7}{|l|}{ COL11A2 } \\
\hline \multicolumn{7}{|l|}{ COL12A1 } \\
\hline \multicolumn{7}{|l|}{ COL13A1 } \\
\hline \multicolumn{7}{|l|}{ COL14A1 } \\
\hline \multicolumn{7}{|l|}{ COL15A1 } \\
\hline \multicolumn{7}{|l|}{ COL16A1 } \\
\hline \multicolumn{7}{|l|}{ COL17A1 } \\
\hline \multicolumn{7}{|l|}{ COL18A1 } \\
\hline COL19A1 & & & & & $0.174^{*} 0.020$ & \\
\hline COL20A1 & & & & & & \\
\hline COL21A1 & & & $-0.163^{* 0.029}$ & & & \\
\hline COL22A1 & & & & & & \\
\hline COL23A1 & & & & & & \\
\hline
\end{tabular}


COL24A1

COL25A1

$0.147^{* 0.049}$

COL26A1 $0.174^{* 0.020}$

$0.206^{* *} 0.006$

COL27A1

$-0.174^{* 0.020}$

COL28A1

1

Peer] reviewing PDF | (2019:03:35949:2:0:NEW 12 Aug 2019) 


\section{Figure 1}

Differential expression analysis of collagen family genes between ESCC and normal tissues.

(A) and (B) Heatmaps of the DEGs in TCGA and GEO in descending order of logFC. The red and blue colors represent high and low expression, respectively. $* P<0.05$; $* * P<0.01$; ${ }^{* * *} P$ $<0.001$. (C) The Venn diagram showing the overlapped DEGs between the two datasets. (DI) Boxplots of three representative up-regulated genes, COL1A1, COL1OA1 and COL11A1 in TCGA and GEO. (J-O) Boxplots of three representative down-regulated genes, COL4A4, COL6A5 and COL14A1 in TCGA and GEO. DEG, differentially expressed gene; FC, fold change. 
A

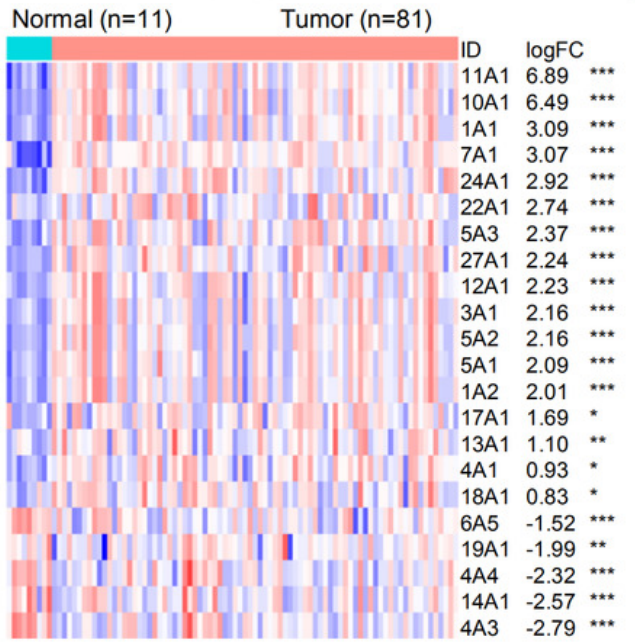

C

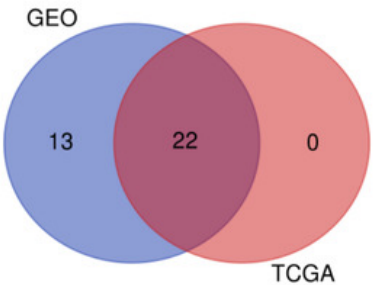

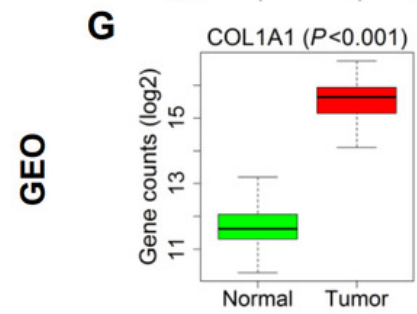
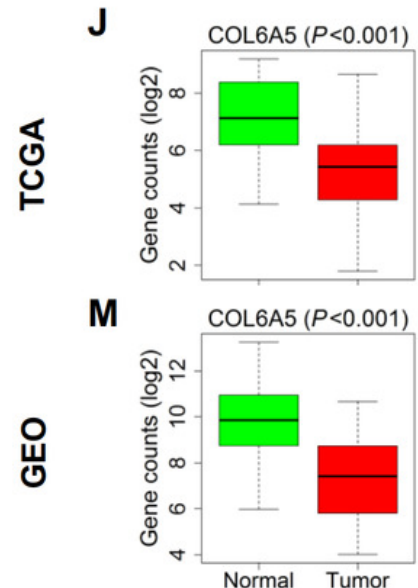

B

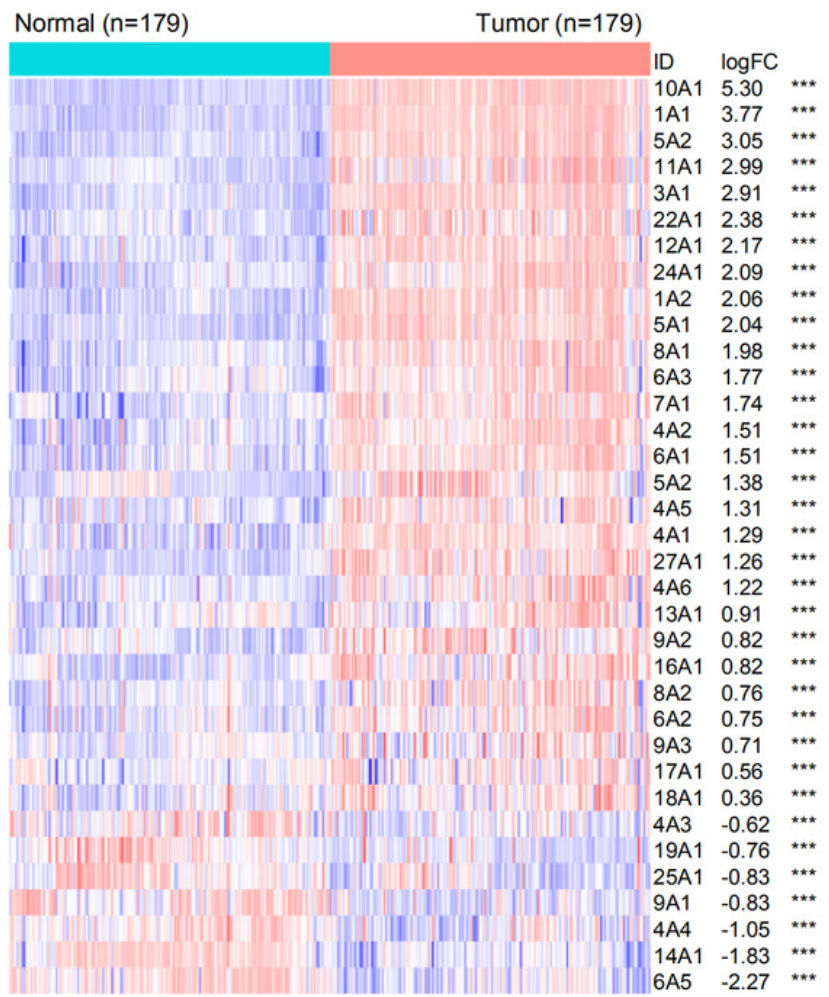

E

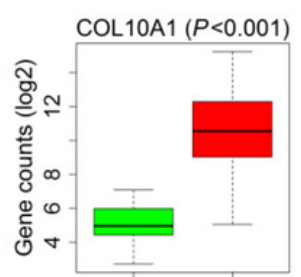

H

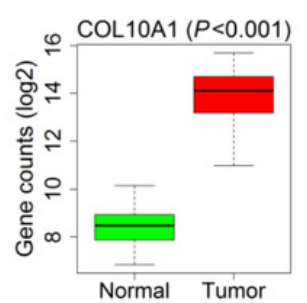

K

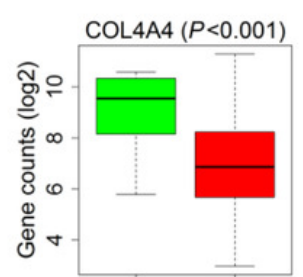

$\mathbf{N}$

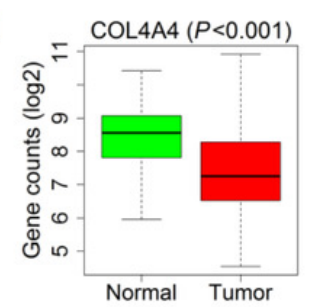

$\mathbf{F}$

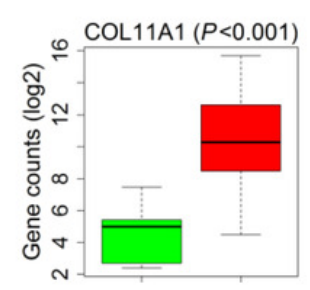

I

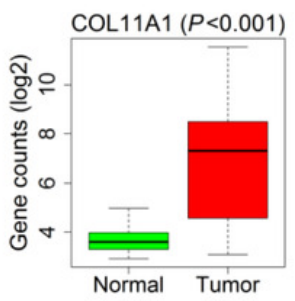

$\mathbf{L}$

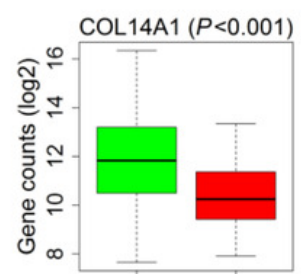

0

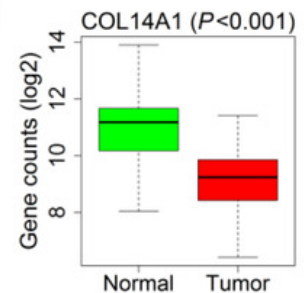


Figure 2

Survival analysis of the DEGs of collagen family in ESCC patients.

(A) and (B) HRs and $P$ values of the DEGs related to overall survival in ascending order of HR in TCGA and GEO. (C) and (D) Kaplan-Meier survival curves of COL6A5 in TCGA and GEO. (E) and (F) Kaplan-Meier survival curves of COL18A1 in TCGA and GEO. DEG, differentially expressed gene; HR, hazard ratio.

A

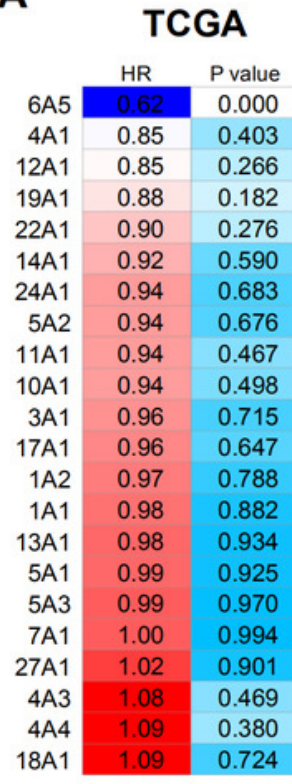

B

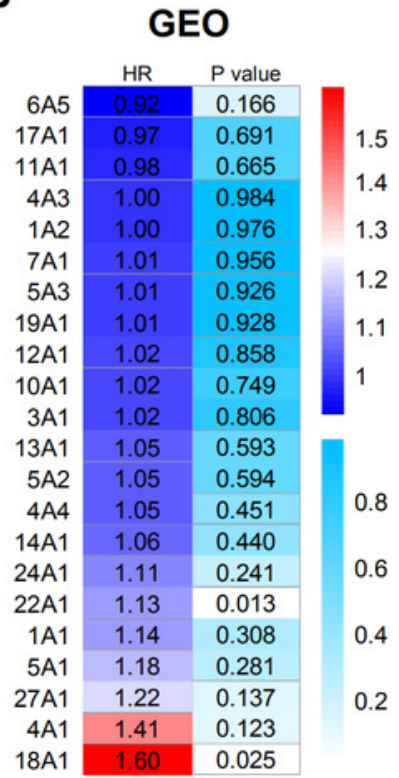

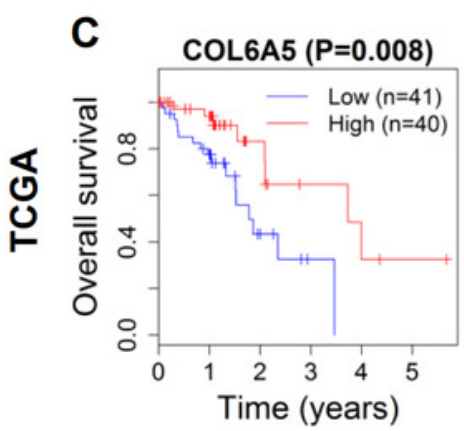

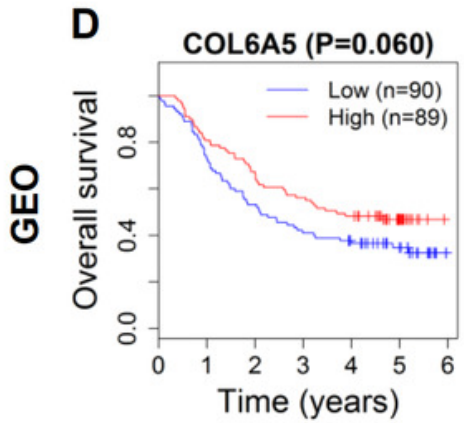

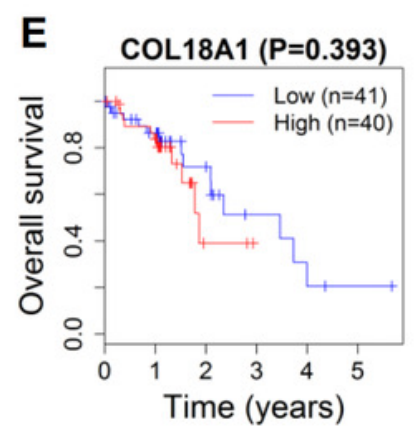

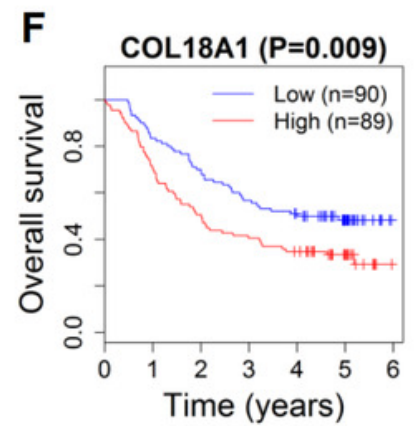




\section{Figure 3}

Prediction models to predict the survival of ESCC patients.

(A-D) ROC and survival curves of the models based on expression of 7 and 9 collagen DEGs respectively in TCGA and GEO. (E-H) ROC and survival curves of the models according to TNM staging in TCGA and GEO. (I) A heatmap showing the expression patterns of the 7 genes driving the prediction model in TCGA. (J) Risk score distribution of the patients in ascending order and divided into low-risk (green) and high-risk (red) in TCGA. (K) Survival time and status of the patients in order of increasing risk scores in TCGA. The red and green dots represent dead and alive, respectively. ROC, receiver operating characteristic; AUC, area under curve; DEG, differentially expressed gene; COL, collagen; TNM, tumor-nodemetastasis. 
TCGA

A

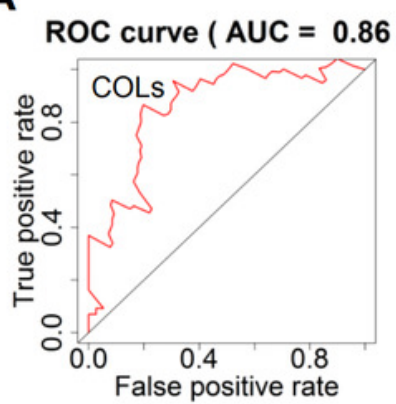

E

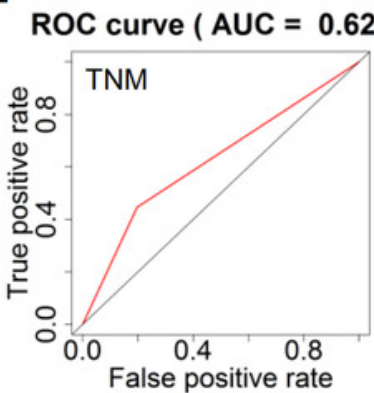

B

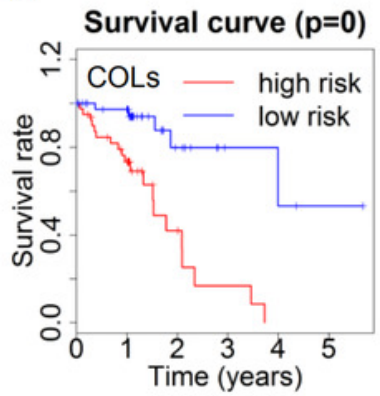

$\mathbf{F}$
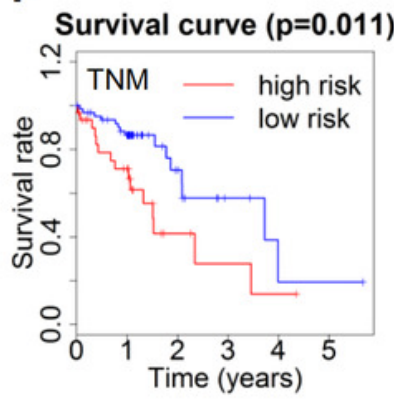

GEO

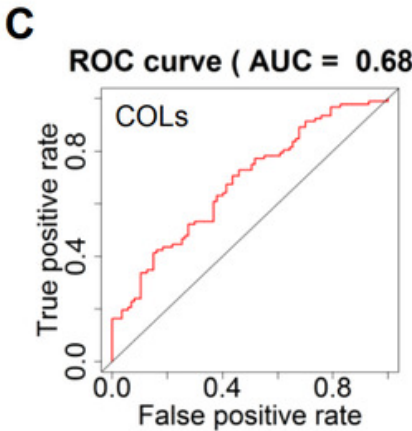

G

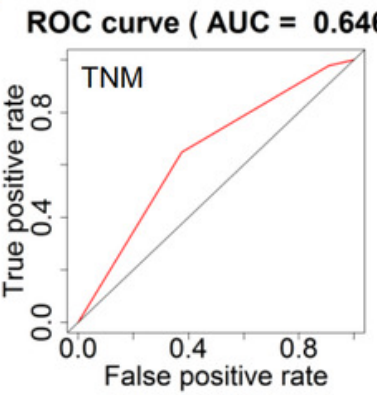

\section{$\mathrm{H}$}

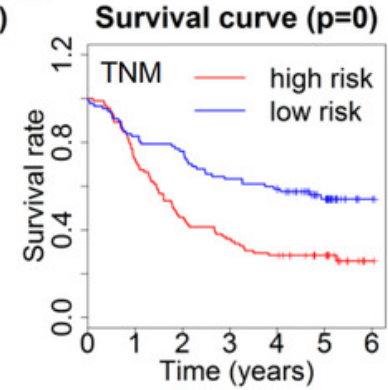

I

Low-risk

High-risk

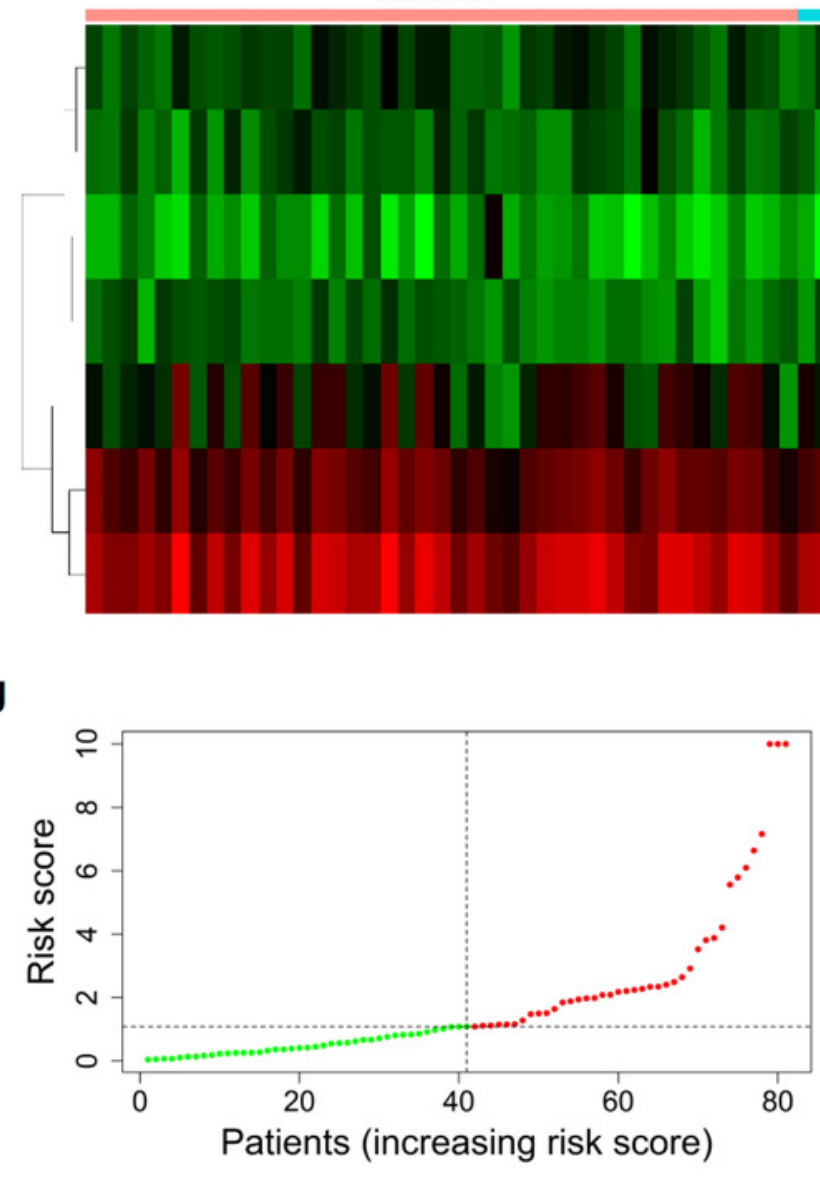

$\mathbf{K}$

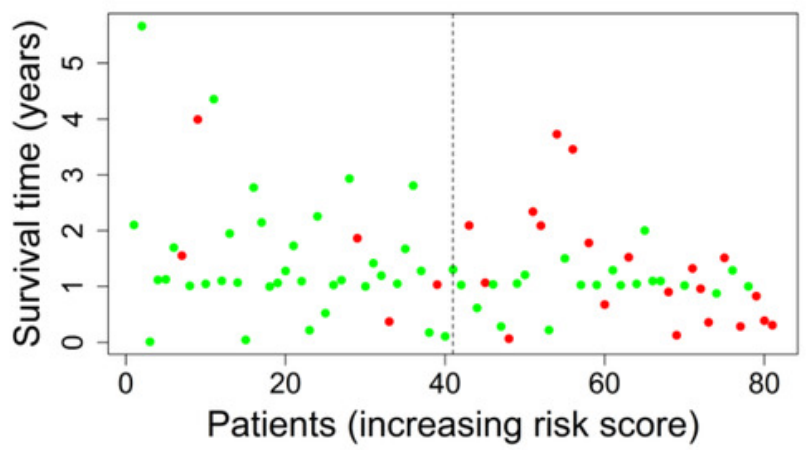


Figure 4

GSEA results based on patient risk scores calculated by the prediction models in TCGA and GEO

(A-F) Representative enriched gene sets according to Oncogenic Signatures Gene Sets. (G-L) Representative enriched gene sets according to Hallmark Gene Sets. (M-R) Representative enriched gene sets according to KEGG Gene Sets. GSEA, gene sets enrichment analysis. NES, normalized enrichment score. 


\section{Oncogenic Signatures Gene Sets}

A

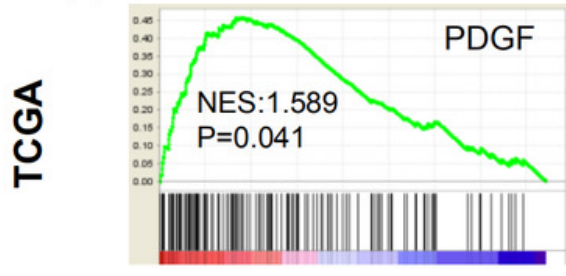

D

잉

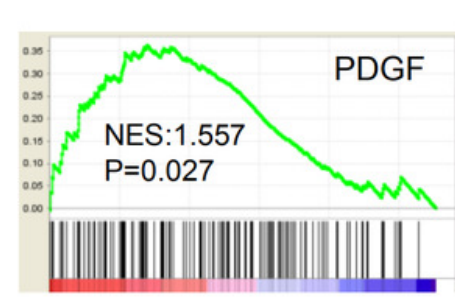

G

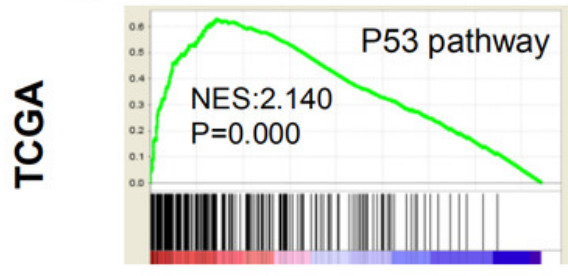

J

입

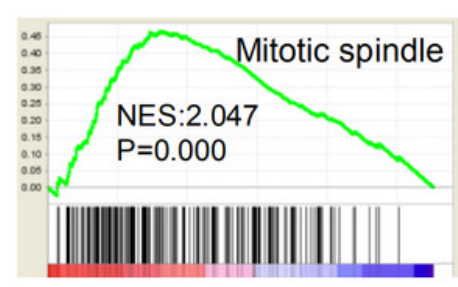

M
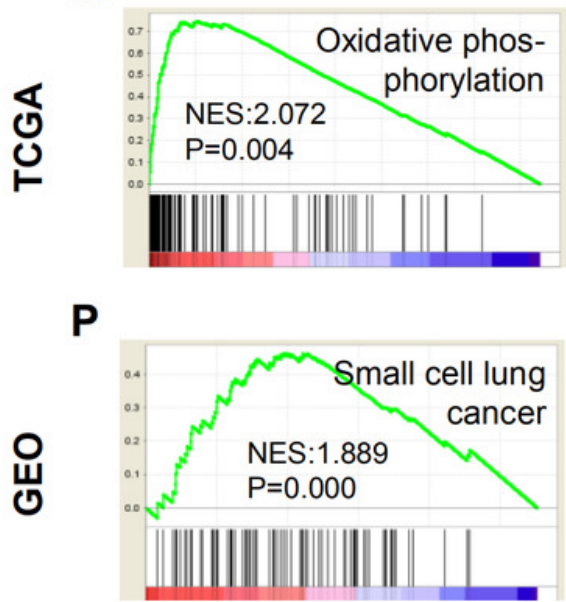

B

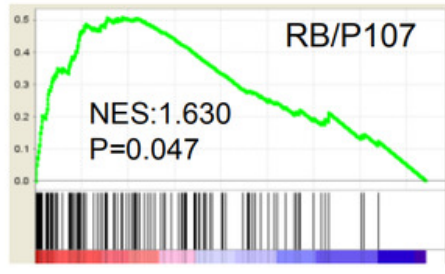

E

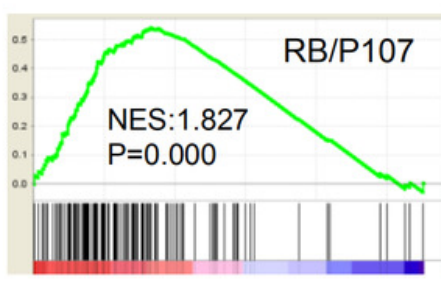

Hallmark Gene Sets

H

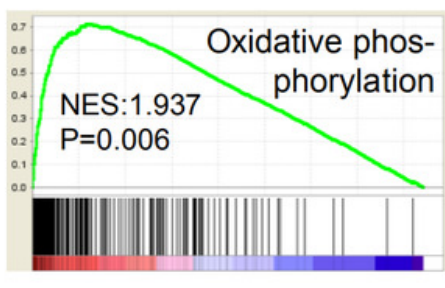

K

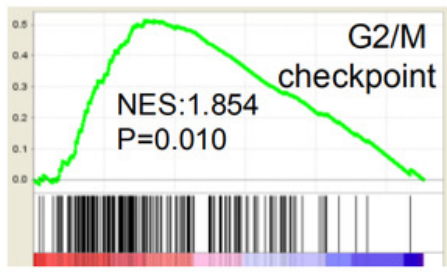

KEGG Gene Sets

N

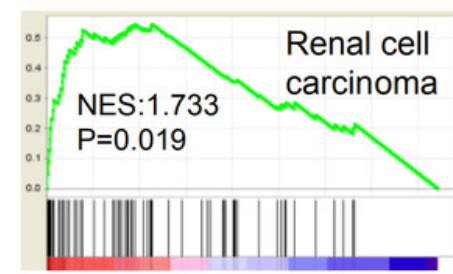

Q

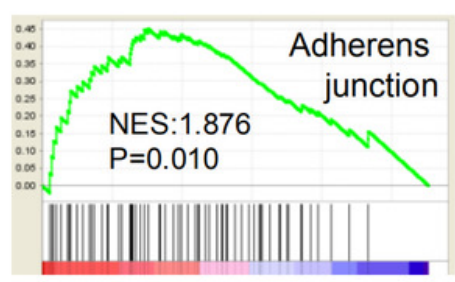

C

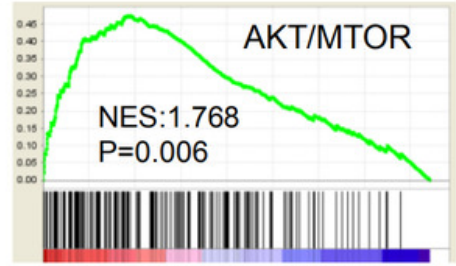

$\mathbf{F}$

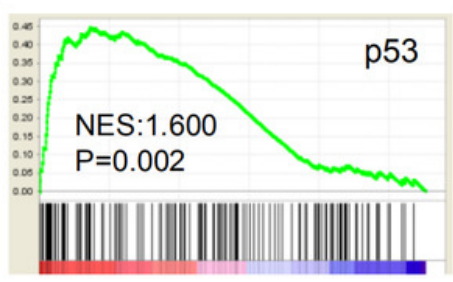

\section{I}

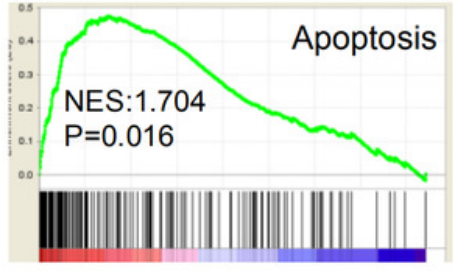

$\mathbf{L}$

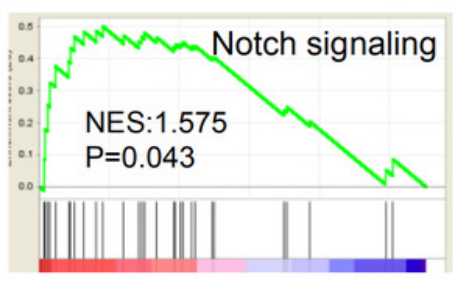

0

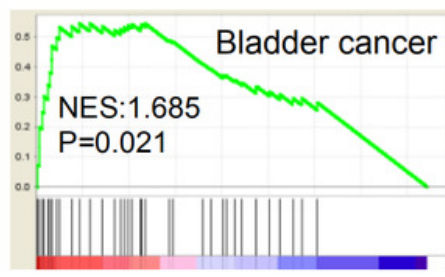

$\mathbf{R}$

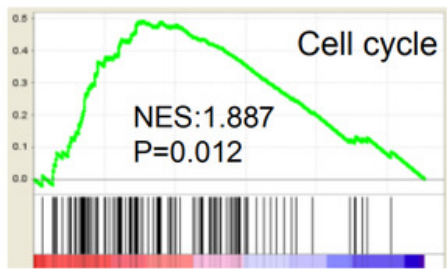




\section{Figure 5}

Co-expression network of collagen family genes.

Visualization of the co-expression between collagen family genes and the risk-scores-based DEGs. The red nodes are collagen family genes, and the bigger ones are the genes included in the 7-gene prediction model in TCGA. The blue nodes are the co-expressed genes. DEG, differentially expressed gene. 


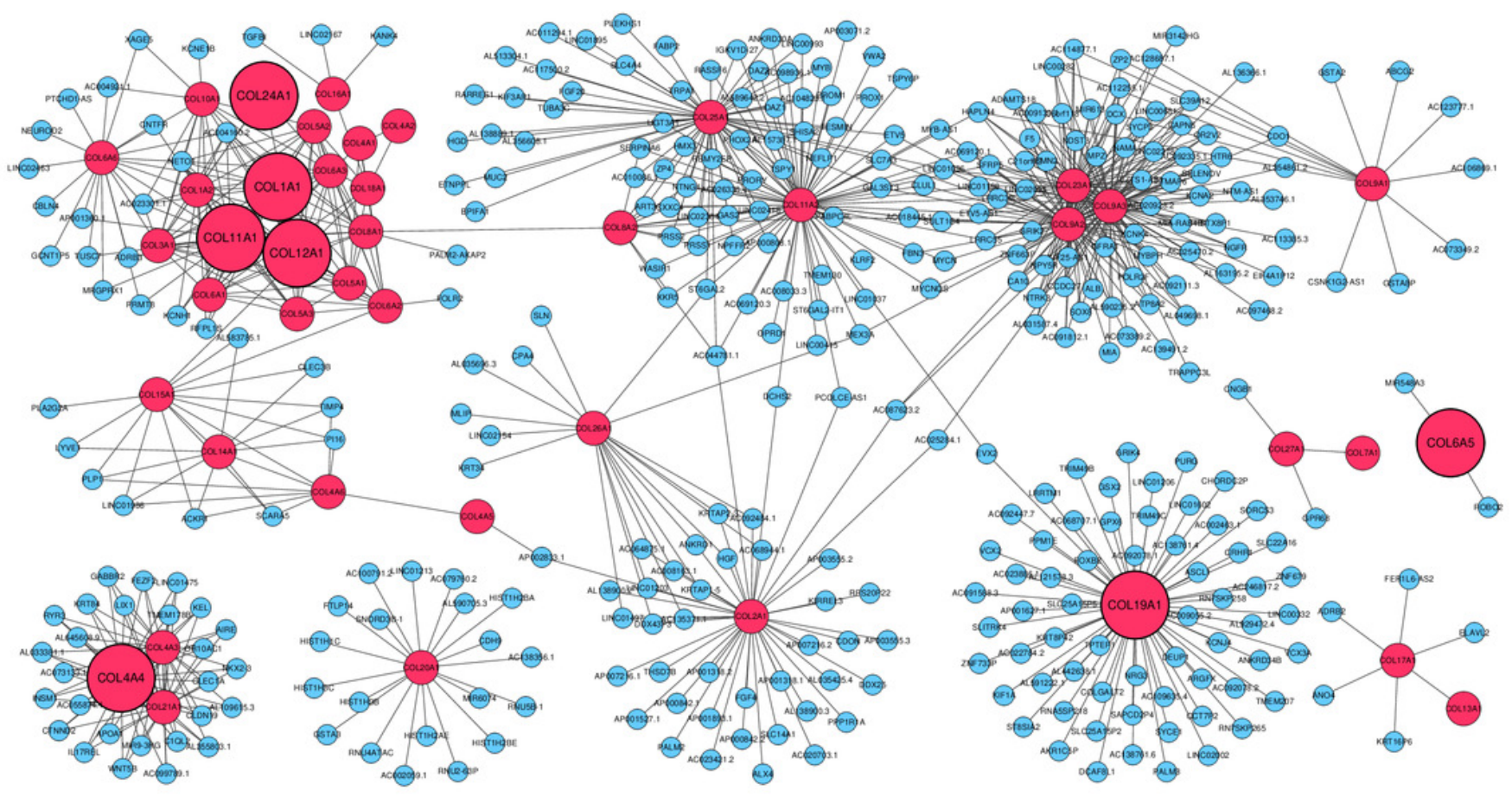

